\title{
The efficacy of peroral cholangioscopy for difficult bile duct stones and indeterminate strictures: a systematic review and meta-analysis
}

Authors

Institutions
Praneet Korrapati ${ }^{1}$, Jody Ciolino ${ }^{1}$, Sachin Wani ${ }^{2}$, Janak Shah ${ }^{3}$, Rabindra Watson ${ }^{4}$, V. Raman Muthusamy ${ }^{4}$, Jason Klapman ${ }^{5}$, Srinadh Komanduri'

Institutions are listed at the end of article. submitted 28. July 2015 accepted after revision 15. December 2015

\section{Bibliography}

Dol http://dx.doi.org/ 10.1055/s-0042-100194 Published online: 4.2.2016 Endoscopy International Open 2016; 04: E263-E275

(c) Georg Thieme Verlag KG Stuttgart . New York E-ISSN 2196-9736

\section{Corresponding author}

\section{Srinadh Komanduri, MD MS}

Division of Gastroenterology and Hepatology

Northwestern University Feinberg School of Medicine 676 St. Clair St., Suite 14-003 Chicago, IL 60611

USA

Fax: +1-312-926-0239

koman1973@gmail.com
License terms

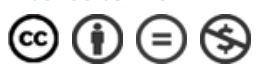

Background and study aims: Current evidence supporting the efficacy of peroral cholangioscopy (POC) in the evaluation and management of difficult bile duct stones and indeterminate strictures is limited. The aims of this systematic review and meta-analysis were to assess the following: the efficacy of POC for the therapy of difficult bile duct stones, the diagnostic accuracy of POC for the evaluation of indeterminate biliary strictures, and the overall adverse event rates for POC.

Patients and methods: Patients referred for the removal of difficult bile duct stones or the evaluation of indeterminate strictures via POC were included. Search terms pertaining to cholangioscopy were used, and articles were selected based on preset inclusion and exclusion criteria. Quality assessment of the studies was completed with a modified Newcastle-Ottawa Scale. After critical literature review, relevant outcomes of interest were analyzed. Meta-regression was performed

\section{Introduction \\ $\nabla$}

During the last several decades, many advances in technology have rendered peroral cholangioscopy (POC) a useful diagnostic and therapeutic technique. POC is conducted during endoscopic retrograde cholangiopancreatography (ERCP) in one of three ways: with a dual-operator dedicated ("mother-daughter") cholangioscopic system, with a single-operator catheter-based cholangioscopic system (SOC), or directly with an ultraslim endoscope or slim gastroscope. The procedures vary with respect to number of operators, maneuverability, image quality, and method of access, resulting in variable success rates.

POC is most commonly used for treating difficult bile duct stones with electrohydraulic lithotripsy or laser lithotripsy or for directly visualizing and/ or sampling indeterminate biliary strictures. Other indications and reported uses for POC include, but are not limited to, placing a guidewire to examine potential sources of between-study variation. Publication bias was assessed via funnel plots and Egger's test.

Results: A total of 49 studies were included. The overall estimated stone clearance rate was $88 \%$ (95\% confidence interval [95\%CI] 85\%-91\%). The accuracy of POC was $89 \%$ (95\%CI $84 \%-93 \%$ ) for making a visual diagnosis and and $79 \%(95 \% \mathrm{Cl}$ $74 \%-84 \%$ ) for making a histological diagnosis. The estimated overall adverse event rate was $7 \%$ (95\%CI 6\%-9\%).

Conclusions: POC is a safe and effective adjunctive tool with endoscopic retrograde cholangiopancreatography (ERCP) for the evaluation of bile duct strictures and the treatment of bile duct stones when conventional methods have failed. Prospective, controlled clinical trials are needed to further elucidate the precise role of POC during ERCP.

during ERCP, monitoring primary sclerosing cholangitis, facilitating stent placement for biliary drainage, assessing the extent of biliary malignancy before surgery, and staging and ablating biliary tumors [1-4]. POC is a safe procedure associated with a low adverse event rate. Variable results have been published in regard to its efficacy and safety for these indications [5]. As such, the aim of this study was to perform a systematic review and meta-analysis to assess (i) the overall clinical efficacy of POC for the therapy of difficult bile duct stones, (ii) the accuracy of POC for diagnosing indeterminate biliary strictures, and (iii) the overall adverse event rate of POC. 


\section{Patients and methods}

$\nabla$

This review and meta-analysis was performed in accordance with the Preferred Reporting Items for Systematic Reviews and MetaAnalysis (PRISMA) statement [6].

Information sources and medical literature search A search for eligible publications was conducted via Ovid Medline, the Cochrane Library, and Scopus with the following key words: cholangiopancreatoscopy, choledochoscopy, pancreatocholangioscopy, cholangioscopy, and pancreatoscopy. Two authors (P.K. and S.K.) independently conducted a medical literature search and screened the resulting studies for inclusion. One reviewer (P.K.) extracted data from all studies that met inclusion criteria and stored relevant data in an Excel (Microsoft, Redmond, Washington, USA) database, and a second reviewer (S.K.) performed a second pass of data entry. A third reviewer (S.W.) resolved any discrepancies. EndNote X7 (Thomson Reuters, New York, New York, USA) was used for reference management.

\section{Eligibility criteria}

For the systematic review, our search included all clinical studies evaluating POC until December 2014.

Inclusion criteria were as follows: (i) studies that investigated POC for the removal of difficult bile duct stones, (ii) studies that investigated POC and its ability to help diagnose indeterminate biliary strictures, (iii) studies that enrolled more than 10 participants, and (iv) full-text articles in English. Notably, difficult bile duct stones were most often defined as stones that could not be removed via conventional methods (ERCP with standard extraction balloons, baskets, or lithotriptors; large endoscopic papillary balloon dilation). Indeterminate biliary strictures were most often defined as strictures that could not be definitively diagnosed with conventional ERCP sampling techniques (brushings, intraductal biopsy).

Exclusion criteria were as follows: (i) case reports, (ii) abstracts, (iii) reviews, (iv) letters to authors or editors, (v) studies evaluating percutaneous cholangioscopy, (vi) animal studies, and (vii) studies evaluating pancreatoscopy only.

\section{Quality assessment}

A modified Newcastle-Ottawa Scale [7] was employed to assess the methodological quality of each study included in this review. The studies were divided into two groups: those in which biliary stone removal was an indication for POC and those in which POC was used for the diagnosis of indeterminate strictures; it should be noted that these two groups of studies are not mutually exclusive.

The scale assessed the following for "Selection" criteria: (i) representativeness of the exposed cohort, (ii) ascertainment of exposure, and (iii) demonstration that the outcome of interest was not present at the start of the study. The scale also assessed the following for "Outcome" criteria: (i) assessment by record linkage; (ii) follow-up length, which was determined to be an average follow-up in the study of at least 6 months for both the evaluation of recurrent stones and clinical follow-up for indeterminate strictures; and (iii) percentage of patients lost to follow-up, which was determined to be less than $15 \%$. Follow-up length and percentage of patients who were lost to follow-up were not used for studies evaluating biliary stone clearance because these factors are not commonly assessed in patients after stone removal.
Thus, according to the modified Newcastle-Ottawa Scale that was used, studies evaluating outcomes of POC for difficult bile duct stones could receive a maximum of four points, and studies evaluating outcomes of POC for indeterminate strictures could receive a maximum of six points. Any question regarding the allocation of points for each study was discussed by three reviewers (P.K., S.K., and S.W.).

\section{List of items and data collected}

The following data elements were extracted (if available) from each study included in the review: (i) publication year; (ii) number of centers involved (single center or multicenter); (iii) setting (university, multicenter, or community); (iv) study design (prospective, retrospective, or randomized controlled trial); (v) type of cholangioscopy (peroral dual-operator dedicated cholangioscope, peroral catheter-based cholangioscope [SpyGlass; Boston Scientific, Natick, Massachusetts, USA], direct peroral cholangioscope or ultraslim endoscope); (vi) study focus (stones, strictures, or both); (vii) sample size; (viii) number of POC procedures attempted; (ix) POC technical success rate (i.e., number of successful POC procedures divided by number attempted POC procedures); (x) adverse event rate; (xi) number of patients lost to follow up; and (xii) follow-up period (mean).

For studies evaluating the outcomes of POC for difficult bile duct stones, additional data included the following: (i) number of patients undergoing stone removal (denominator for stone clearance rate); (ii) stone clearance rate (rate of complete stone clearance, not including partial clearance); (iii) average number of stones per patient (mean); (iv) average stone size in millimeters (mean); (v) location of more than $75 \%$ of stones (extrahepatic, intrahepatic, cystic, or mixed); (vi) stone removal technique (cholangioscopy-assisted basket or balloon, electrohydraulic lithotripsy, laser lithotripsy, or multiple methods); and (vii) stone recurrence rate.

For studies in which the outcomes of POC for indeterminate strictures were determined by visual impression only, additional relevant data included the following: (i) number of patients involved in the diagnostic study (denominator for accuracy), (ii) number of patients with true malignant disease (denominator for sensitivity), (iii) number of patients with true benign disease (denominator for specificity), (iv) sensitivity, (v) specificity, (vi) positive predictive value, (vii) negative predictive value, and (viii) accuracy. For studies in which the outcomes of POC for indeterminate strictures were determined by directed tissue sampling, additional relevant data included the following: (i) number of patients or biopsy samples involved in the diagnostic study (denominator for accuracy), (ii) mean number of biopsy samples per patient/ procedure, (iii) number of patients with true malignant disease (denominator for sensitivity), (iv) number of patients with true benign disease (denominator for specificity), (v) sensitivity, (vi) specificity, (vii) positive predictive value, (viii) negative predictive value, and (ix) accuracy.

\section{Outcomes measured}

The primary outcomes for studies evaluating POC for difficult bile duct stone included the following: (i) technical success rate (ability to achieve selective bile duct access), (ii) stone clearance rate, and (iii) stone recurrence rate. The primary outcomes for studies evaluating POC for indeterminate strictures included the following: (i) technical success rate (ability to achieve selective bile duct access), (ii) accuracy (both visual and directed tissue sampling), (iii) sensitivity (both visual and directed tissue sampling), and 


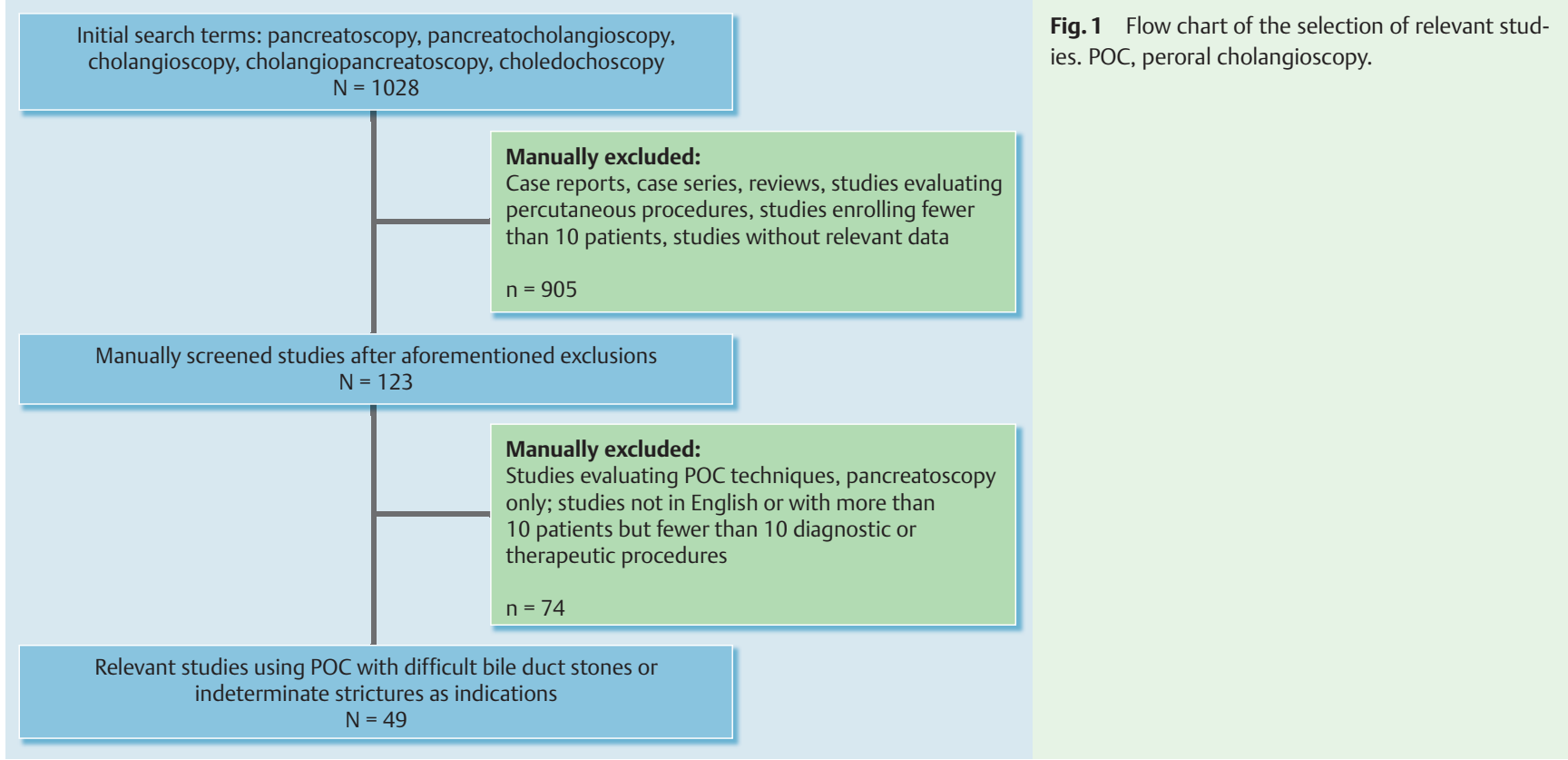

(iv) specificity (both visual and directed tissue sampling). The overall adverse event rate related to POC was determined.

\section{Statistical analysis and summary measures}

Comprehensive Meta-Analysis Software v2.0 (Biostat, Englewood, New Jersey, USA) was used for all formal meta-analyses (when the number of studies was more than five) to obtain summary estimates of proportions (stone clearance rate, technical success rates, stone recurrence rate, adverse event rates, sensitivities, specificities, and accuracy rates). Because of the assumption of inherently different study scenarios and study populations, a random effects model for all analyses was assumed. Heterogeneity across studies via a chi-squared test on the Q-statistic with appropriate degrees of freedom (dependent on outcome because not all studies uniformly reported all outcomes of interest) and the estimated measure of excess-to-total variation $\left(I^{2}\right)$ across studies for each outcome of interest were also calculated. In instances in which the degrees of freedom were sufficiently large and there was significant evidence of between-study variation (i.e., heterogeneity), meta-regression to examine potential sources of between-study variation was performed.

Publication bias was assessed via funnel plots and Egger's test on the regression intercept for these plots. In instances of significant evidence of publication bias $(P<0.05)$, imputed studies were used to create adjusted summary estimates for each measure. Other factors, such as differences in trial quality and true study heterogeneity, could produce asymmetry in funnel plots.

\section{Results}

\section{Literature search and included studies}

The outlined search strategy resulted in the identification of a total of 1028 studies. Based on the defined inclusion and exclusion criteria, a total of 49 studies [ $8-56$ ] were included in the analysis (ه Fig. 1). Of the 49 studies evaluated, 33 contained data on difficult bile duct stones ( $\Theta$ Table 1 ) and 29 studies contained data on indeterminate strictures ( $\bullet$ Table 2 ); there were 20 studies fo- cusing only on difficult bile duct stones, 16 studies only on indeterminate strictures, and 13 studies on both.

\section{Efficacy of peroral cholangioscopy for difficult bile duct stones}

The overall estimated stone clearance rate $(\mathrm{n}=31$ studies) was $88 \%$ (95\% confidence interval [95CI] 85\%-91\%), without significant evidence of heterogeneity $\left(P=0.09, I^{2}=26.14\right)$ ( $\bullet$ Fig. 2 ). There was evidence of publication bias $(P=0.0466)$ in this analysis. Imputed values would fall below the estimated mean rate with larger standard errors, and the adjusted stone clearance rate according to the trim and fill method of Duval and Tweedie [57] is $85 \%$ (95\%CI $82 \%-88 \%)$. Study year, study design, stone size, stone location, number of stones, and type of POC had no impact on stone clearance rates based on meta-regression analysis with regard to stone clearance.

The estimated stone recurrence rate ( $\mathrm{n}=6$ studies) was $13 \%(95 \%$ CI $7 \%-20 \%$ ) ( $\odot$ Fig. 3 ) with no evidence of heterogeneity $(P=$ $\left.0.13, I^{2}=40.09\right)$ or publication bias $(P=0.55)$. The estimated technical success rate $(\mathrm{n}=15$ studies) was $91 \%(95 \%$ CI $88 \%-94 \%)$ ( $\bullet$ Fig. 4), with evidence of heterogeneity $\left(P<0.01, I^{2}=61.72\right)$. Meta-regression identified a significant association between the type of POC used and technical success rates, with SOC demonstrating higher technical success rates compared with other methods $(P<0.01)(\bullet$ Fig. 5$)$.

\section{Efficacy of peroral cholangioscopy for indeterminate strictures}

The diagnostic characteristics of POC for visual impression were as follows ( $\bullet$ Table 3$)$ : accuracy ( $\mathrm{n}=10$ studies), $89 \%(95 \% \mathrm{Cl}$ $84 \%-93 \%$ ) ( Fig.6); sensitivity ( $\mathrm{n}=9$ studies), $93 \%$ (95\%Cl $85 \%-97 \%$ ); specificity ( $\mathrm{n}=9$ studies), $85 \%$ (95\%CI 79\%-89\%). In each case, there was no significant evidence of heterogeneity. The diagnostic characteristics of POC for directed tissue sampling were as follows ( $\bullet$ Table 3 ): accuracy ( $\mathrm{n}=13$ studies), $79 \%$ (95\% CI $74 \%-84 \%$ ) ( $\bullet$ Fig.7); sensitivity ( $\mathrm{n}=12$ studies), $69 \%$ (95\%Cl $57 \%-78 \%$ ); specificity ( $\mathrm{n}=10$ studies), $94 \%$ (95\%Cl 89\%-97\%). Meta-regression identified a significant association between the type of POC used and visual accuracy $(P<0.01)$ and between the 


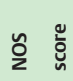

II

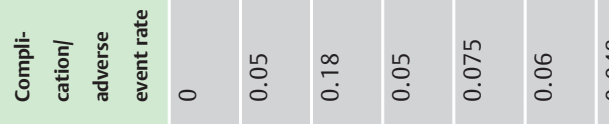

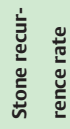

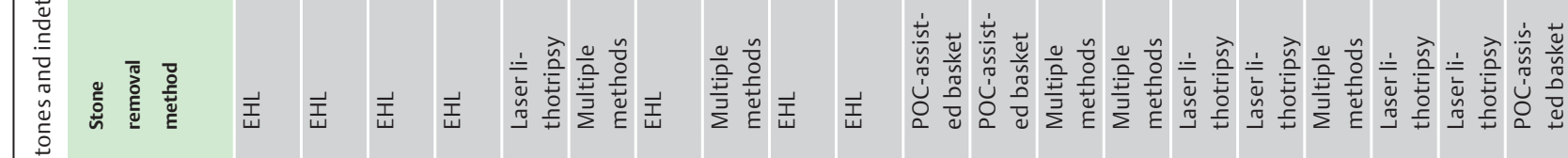

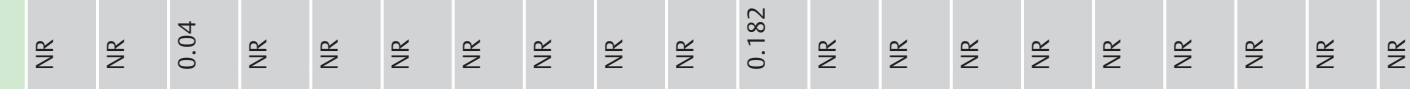

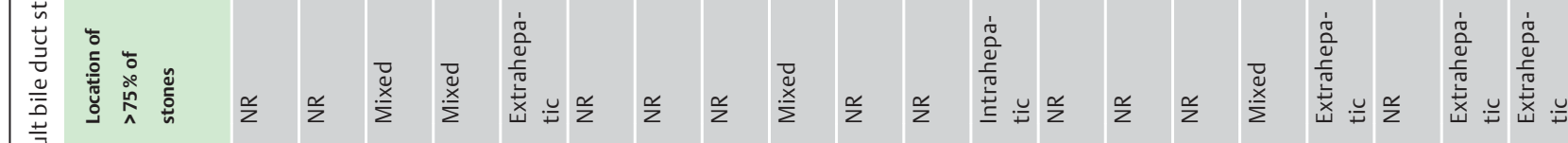

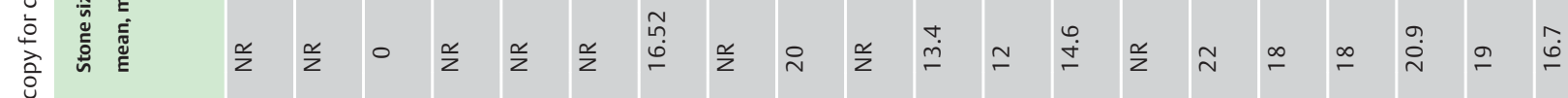

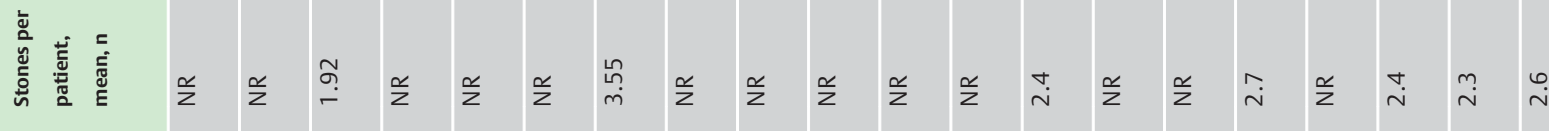
畜

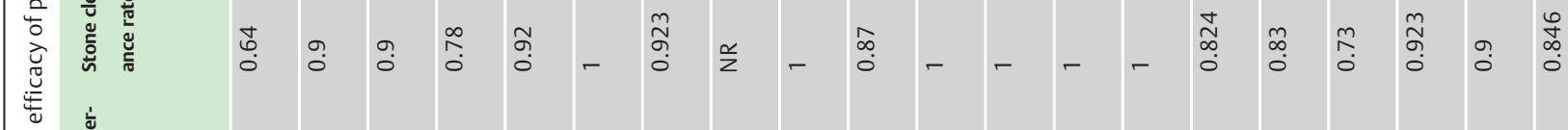

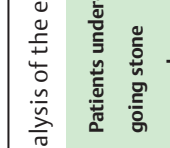

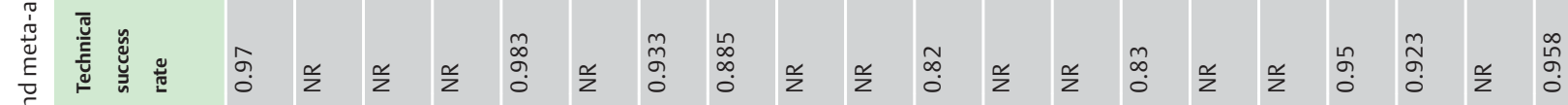

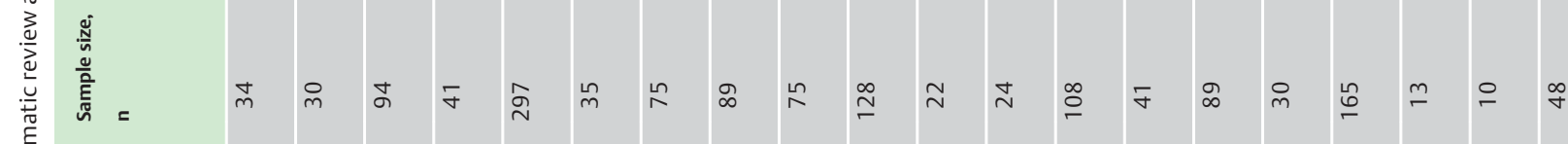

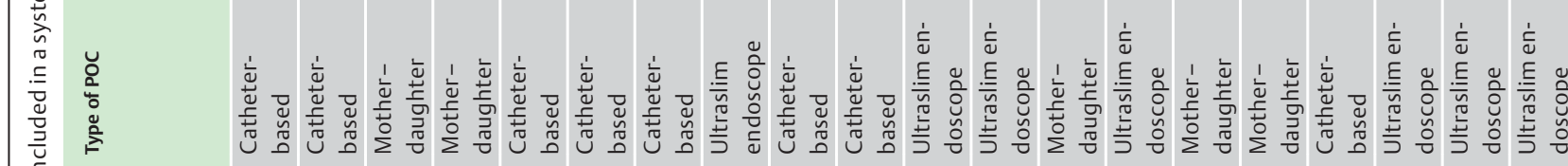

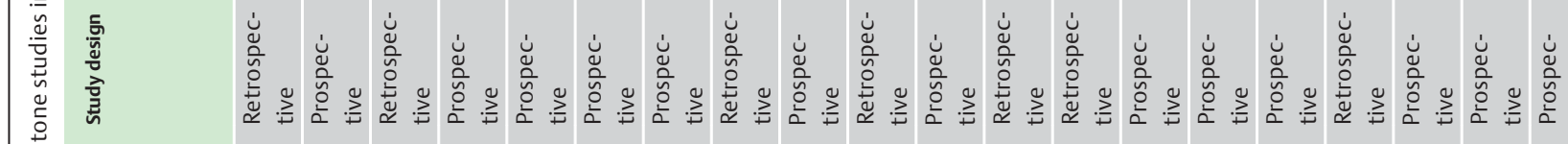

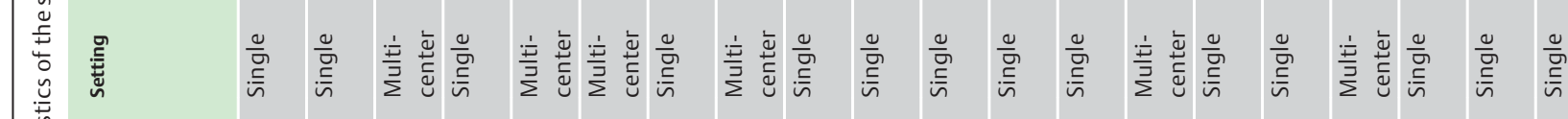

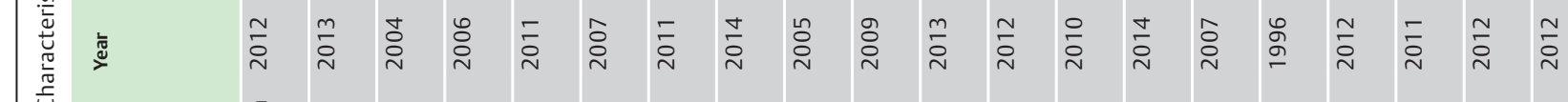

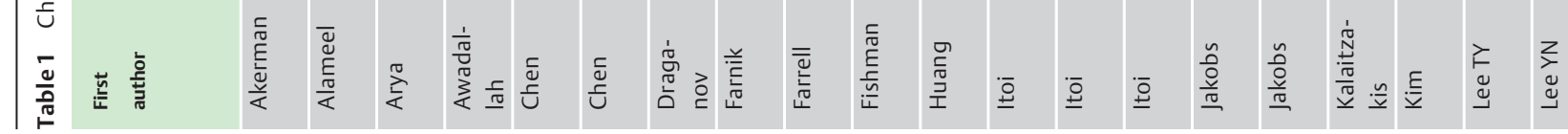




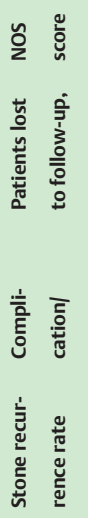

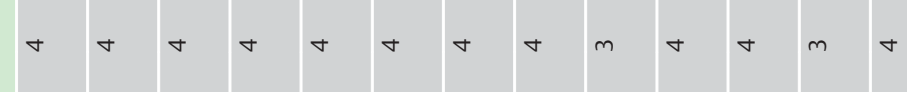

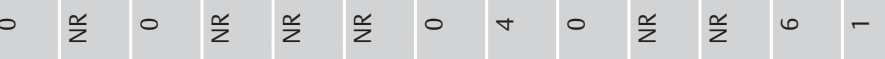

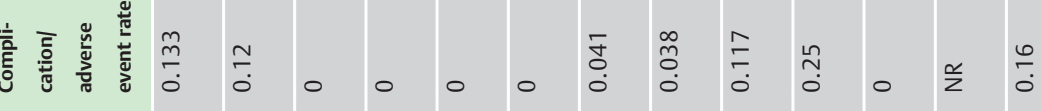

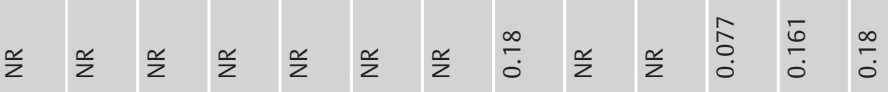

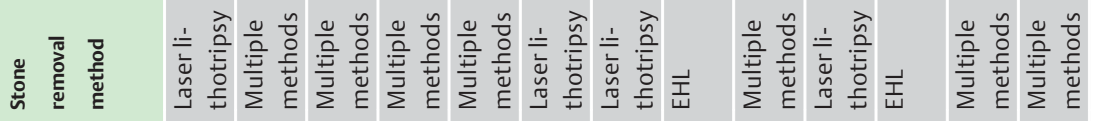

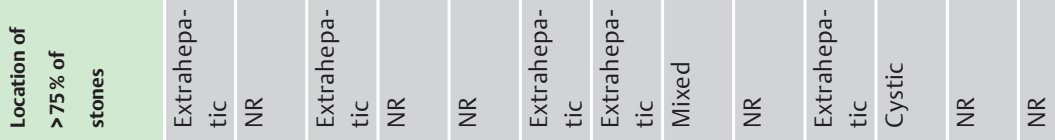

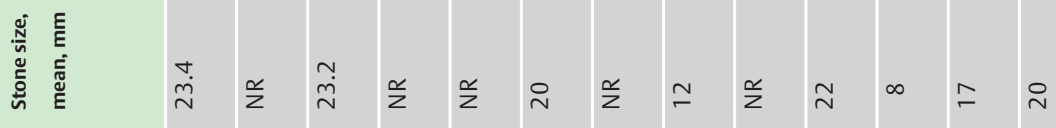

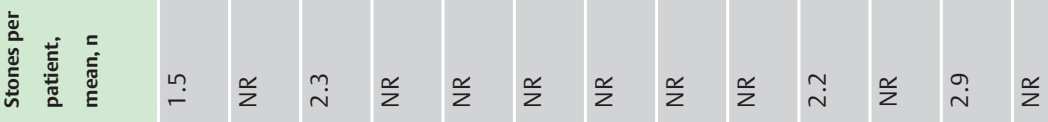

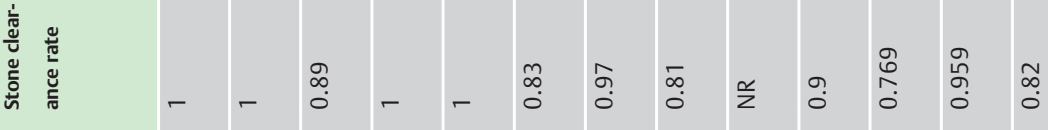

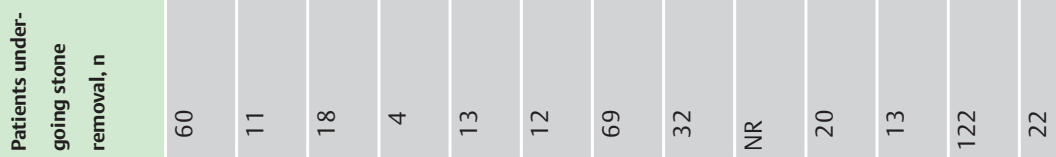

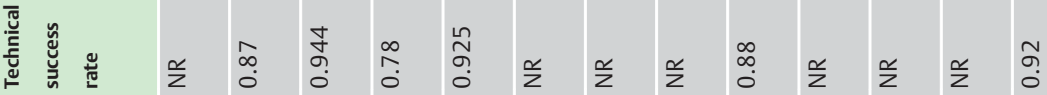

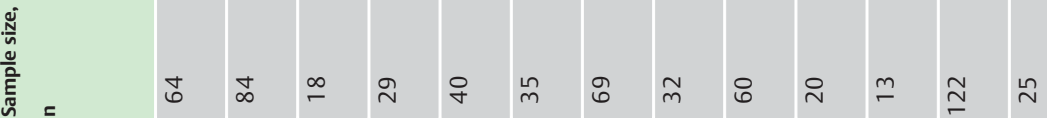

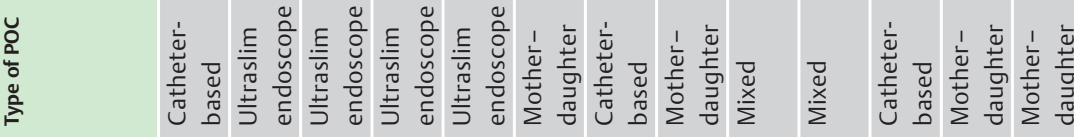

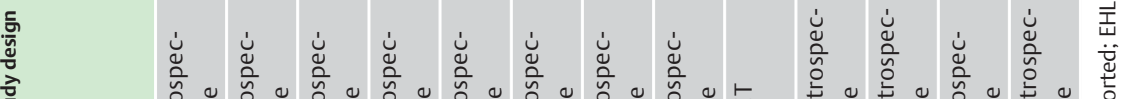

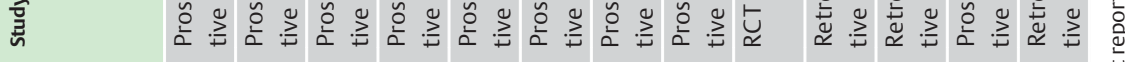

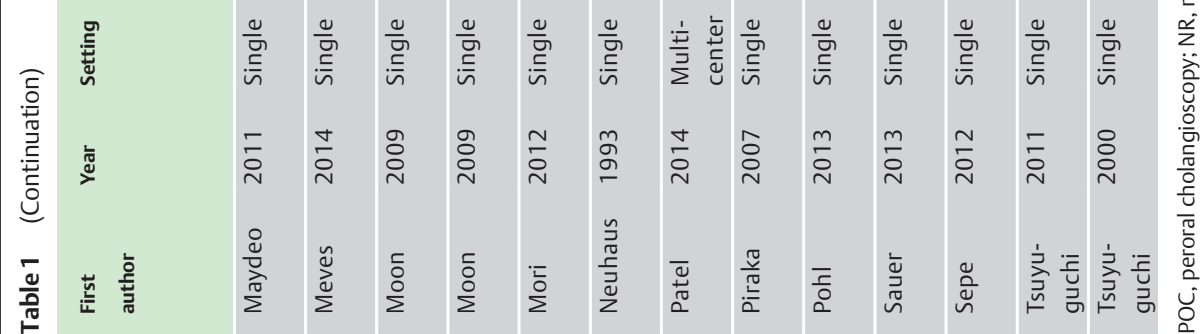


荃萦

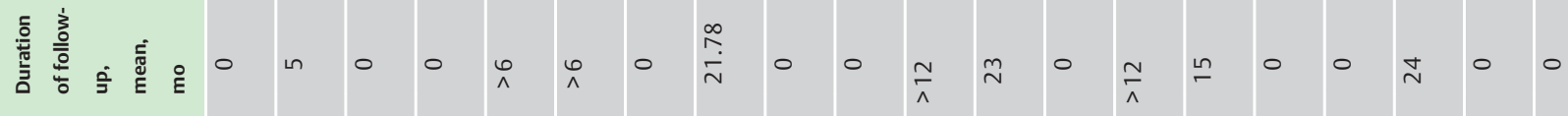

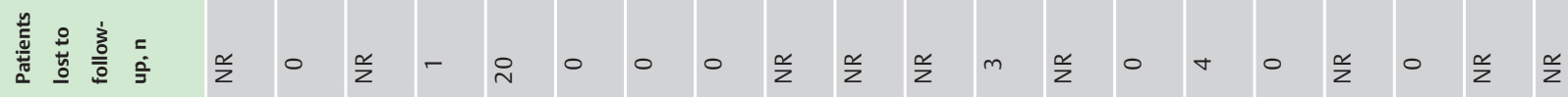

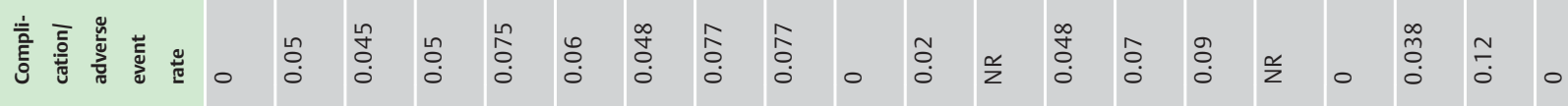

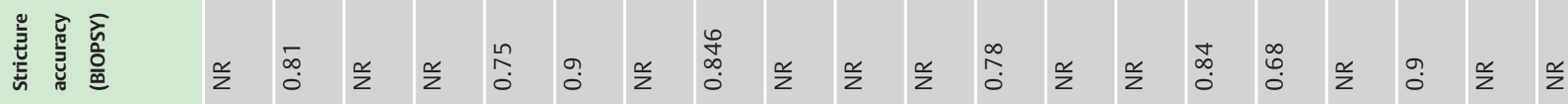

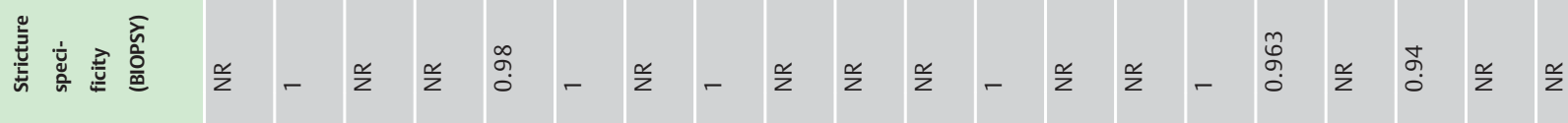

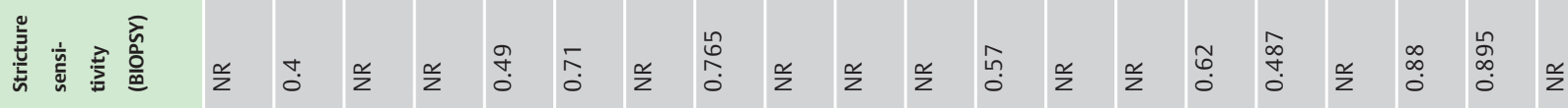

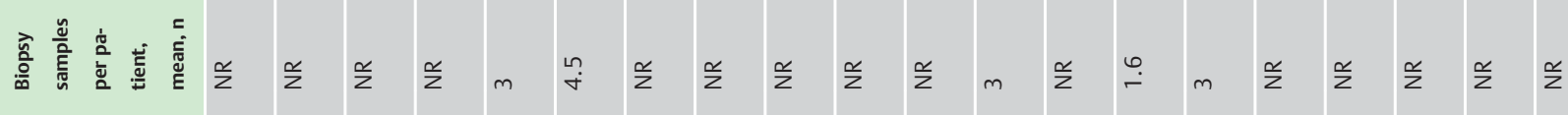

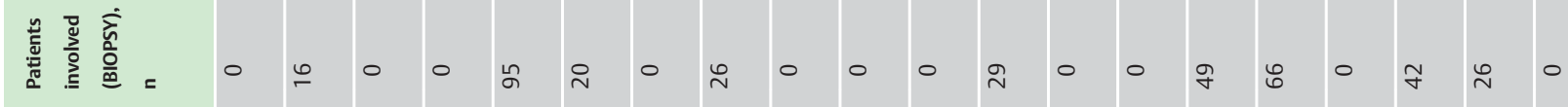

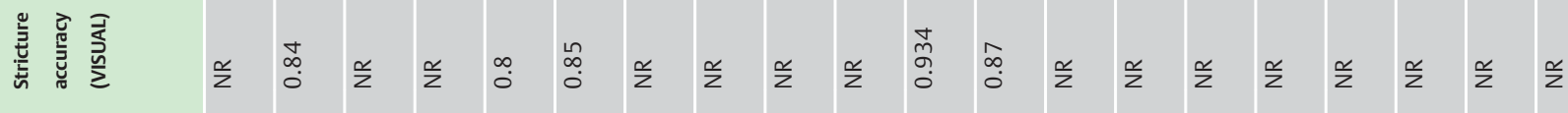

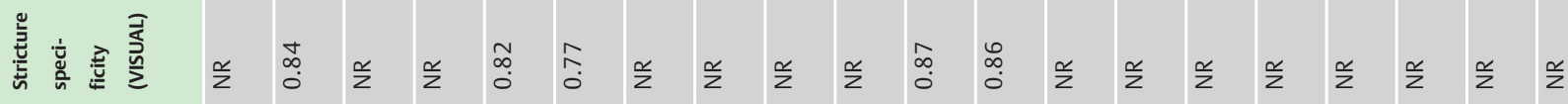

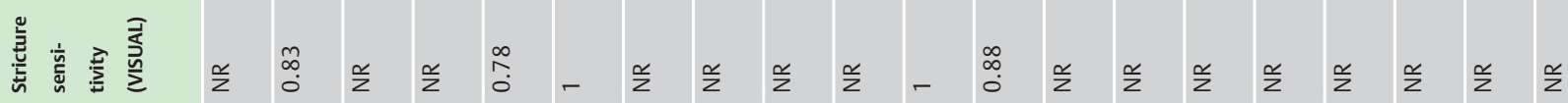

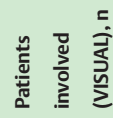

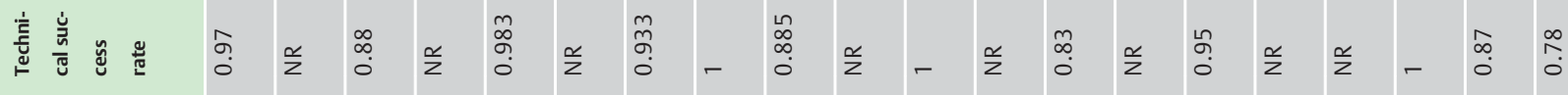

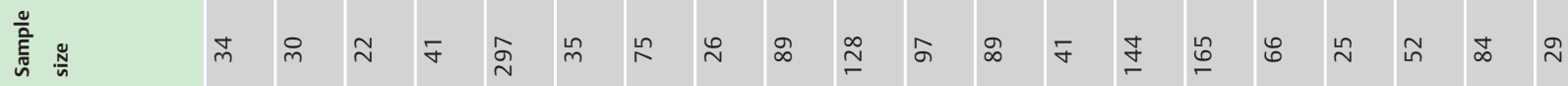

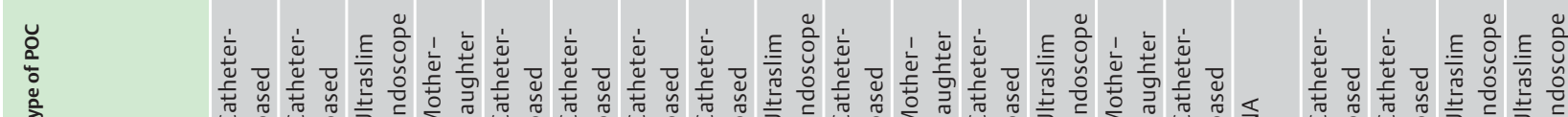

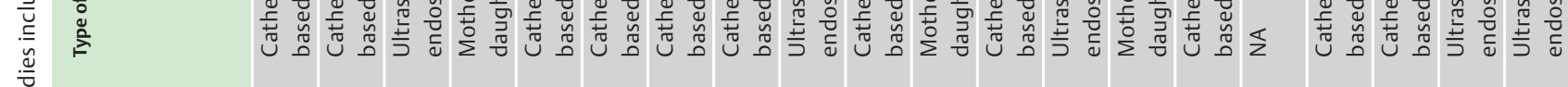

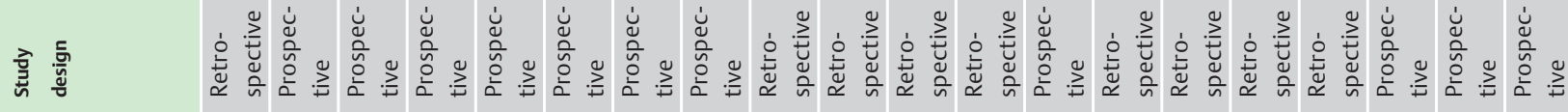

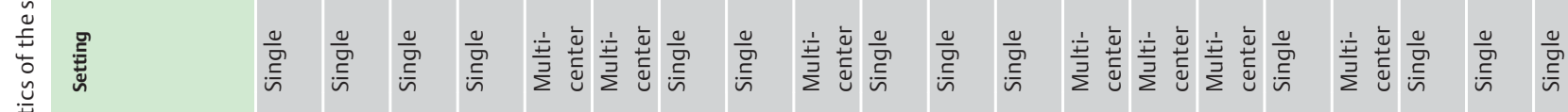

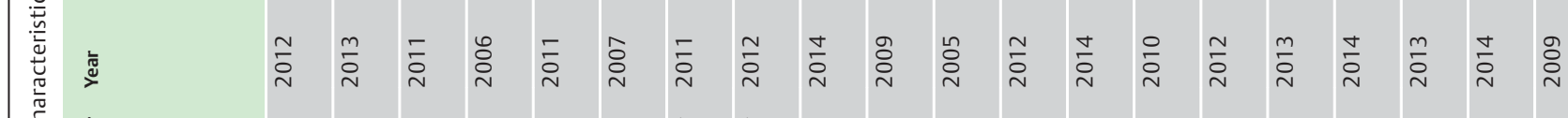

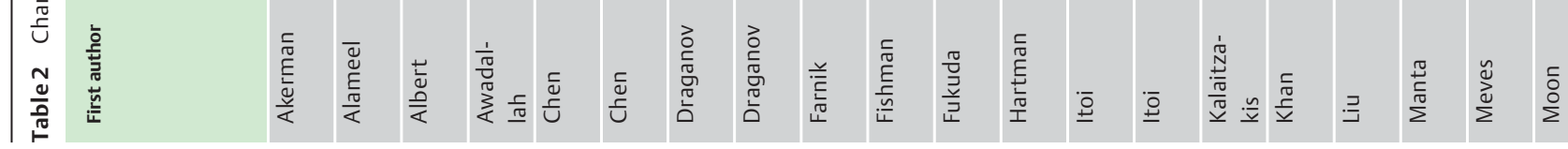




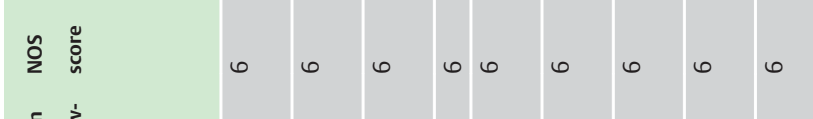

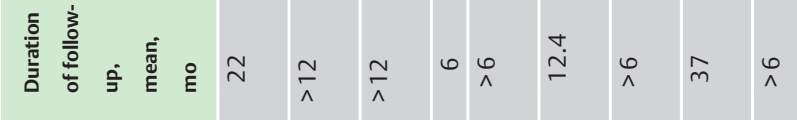

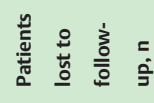

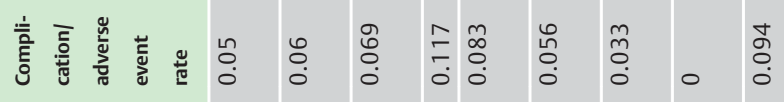

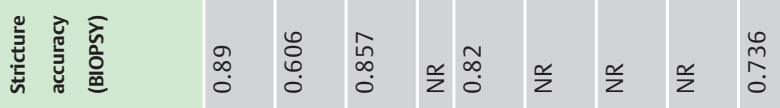

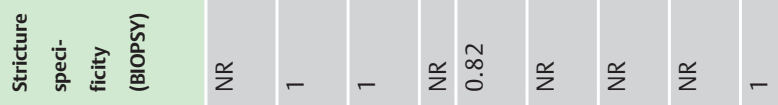

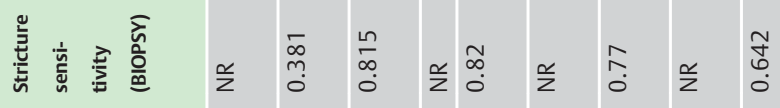

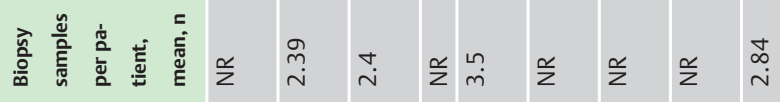

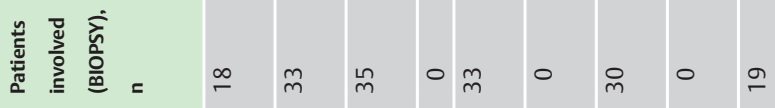

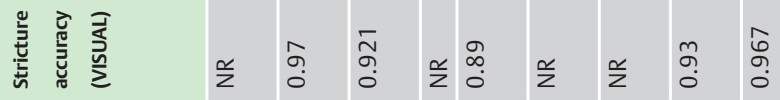

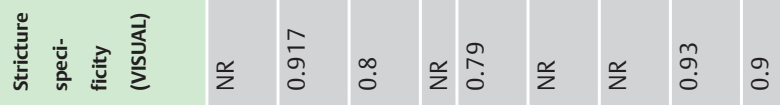

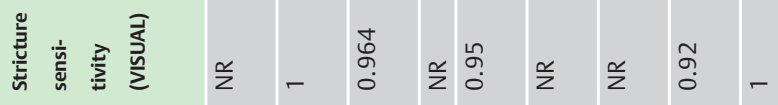

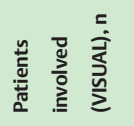

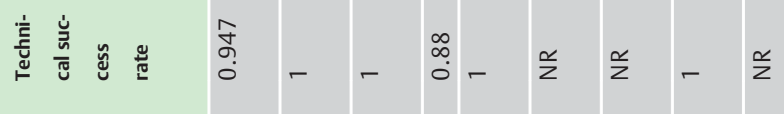

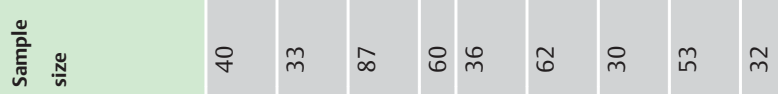

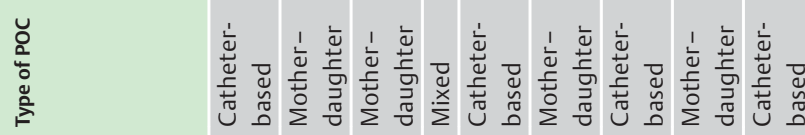

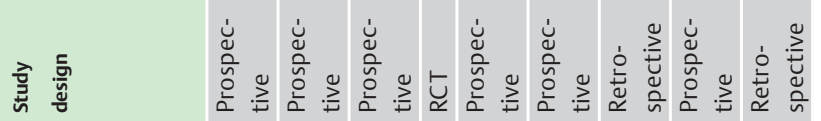

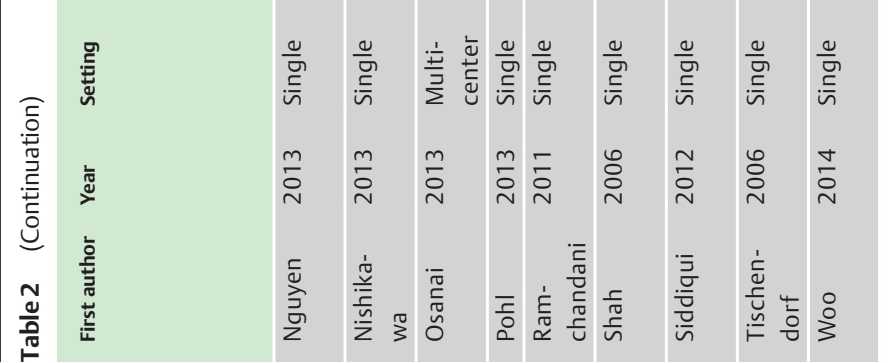


Original article

\begin{tabular}{|c|c|c|c|c|c|}
\hline \multirow[t]{2}{*}{ Study } & \multicolumn{3}{|c|}{ Statisitics for each study } & \multirow[t]{2}{*}{ Event rate and $95 \% \mathrm{Cl}$} & \multirow{3}{*}{$\begin{array}{l}\text { Fig. } 2 \text { Forest plot of studies reporting bile duct } \\
\text { stone clearance rate with peroral cholangioscopy } \\
\text { Pooled clearance rate was } 88 \% \text { ( } 95 \% \text { confidence } \\
\text { interval [CI] } 85 \%-91 \%) \text {. }\end{array}$} \\
\hline & $\begin{array}{l}\text { Event } \\
\text { rate }\end{array}$ & $\begin{array}{l}\text { Lower } \\
\text { limit }\end{array}$ & $\begin{array}{l}\text { Upper } \\
\text { limit }\end{array}$ & & \\
\hline Patel, 2014 & 0.970 & 0.890 & 0.992 & & \\
\hline Sauer, 2014 & 0.900 & 0.676 & 0.975 & & \\
\hline Huang, 2013 & 0.917 & 0.378 & 0.995 & & \\
\hline Mori, 2012 & 0.964 & 0.616 & 0.998 & & \\
\hline Lee, TY, 2012 & 0.900 & 0.533 & 0.986 & & \\
\hline Itoi, 2012 & 0.944 & 0.495 & 0.997 & & \\
\hline Lee, YN, 2012 & 0.846 & 0.549 & 0.961 & & \\
\hline Sepe, 2011 & 0.769 & 0.478 & 0.924 & & \\
\hline Kim, 2011 & 0.923 & 0.609 & 0.989 & & \\
\hline Maydeo, 2011 & 0.992 & 0.882 & 0.999 & & \\
\hline Tsuyuguchi, 2011 & 0.959 & 0.905 & 0.983 & & \\
\hline Itoi, 2010 & 0.981 & 0.764 & 0.999 & & \\
\hline Moon, 2009a & 0.890 & 0.649 & 0.973 & & \\
\hline Jakobs, 2007 & 0.824 & 0.573 & 0.942 & & \\
\hline Piraka, 2007 & 0.810 & 0.638 & 0.912 & & \\
\hline Farrell, 2007 & 0.981 & 0.764 & 0.999 & & \\
\hline Arya, 2004 & 0.900 & 0.821 & 0.946 & & \\
\hline Tsuyuguchi, 2000 & 0.820 & 0.606 & 0.931 & & \\
\hline Jakobs, 1996 & 0.830 & 0.484 & 0.962 & & \\
\hline Neuhaus, 1993 & 0.830 & 0.520 & 0.957 & & \\
\hline Itoi, 2014 & 0.944 & 0.495 & 0.997 & & \\
\hline Meves, 2014 & 0.958 & 0.575 & 0.997 & & \\
\hline Alameel, 2013 & 0.900 & 0.533 & 0.986 & & \\
\hline Akerman, 2012 & 0.640 & 0.342 & 0.859 & & \\
\hline Kalaitzakis, 2012 & 0.730 & 0.556 & 0.854 & & \\
\hline Draganov, 2011 & 0.923 & 0.739 & 0.981 & & \\
\hline Chen, 2011 & 0.920 & 0.825 & 0.965 & & \\
\hline Moon, 2009b & 0.900 & 0.326 & 0.994 & & \\
\hline Fishman, 2009 & 0.870 & 0.729 & 0.943 & & \\
\hline Chen, 2007 & 0.950 & 0.525 & 0.997 & & \\
\hline Awadallah, 2006 & 0.780 & 0.423 & 0.945 & -1 & \\
\hline Summary Rate & 0.885 & 0.850 & 0.912 & 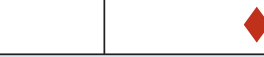 & \\
\hline Prediction Interva & & & & 0.50 & \\
\hline
\end{tabular}

\begin{tabular}{|c|c|c|c|c|c|}
\hline \multirow[t]{2}{*}{ Study } & \multicolumn{3}{|c|}{ Statisitics for each study } & \multicolumn{2}{|c|}{ Event rate and $95 \% \mathrm{Cl}$} \\
\hline & $\begin{array}{l}\text { Event } \\
\text { rate }\end{array}$ & $\begin{array}{l}\text { Lower } \\
\text { limit }\end{array}$ & $\begin{array}{l}\text { Upper } \\
\text { limit }\end{array}$ & & \\
\hline Huang, 2013 & 0.182 & 0.022 & 0.683 & & - \\
\hline Sepe, 2012 & 0.077 & 0.011 & 0.391 & - & \\
\hline Tsuyuguchi, 2011 & 0.161 & 0.106 & 0.237 & & \\
\hline Piraka, 2007 & 0.180 & 0.082 & 0.351 & & \\
\hline Arya, 2004 & 0.040 & 0.015 & 0.105 & & \\
\hline Tsuyuguchi, 2000 & 0.180 & 0.069 & 0.394 & & \\
\hline Summary Rate & 0.128 & 0.078 & 0.204 & 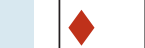 & \\
\hline Prediction Interval & & & & 0.00 & 1.00 \\
\hline
\end{tabular}

Fig. 3 Forest plot of studies reporting stone recurrence rate after clearance by peroral cholangioscopy. Pooled recurrence rate was 13\% (95\% confidence interval $[\mathrm{Cl}] 7 \%-20 \%$ ).

\begin{tabular}{llll|l|l} 
Study & \multicolumn{5}{c}{ Event rate and 95\% Cl } \\
\hline & $\begin{array}{l}\text { Event } \\
\text { rate }\end{array}$ & $\begin{array}{l}\text { Lower } \\
\text { limit }\end{array}$ & $\begin{array}{l}\text { Upper } \\
\text { limit }\end{array}$ \\
Huang, 2013 & 0.820 & 0.606 & 0.931 & & \\
Mori, 2012 & 0.925 & 0.792 & 0.976 & & \\
Lee, YN, 2012 & 0.958 & 0.848 & 0.989 & & \\
Kim, 2011 & 0.923 & 0.609 & 0.989 & & \\
Moon, 2009a & 0.944 & 0.693 & 0.992 & \\
Tsuyuguchi, 2000 & 0.920 & 0.731 & 0.980 & \\
Itoi, 2014 & 0.830 & 0.684 & 0.917 & \\
Farnik, 2014 & 0.885 & 0.818 & 0.930 & \\
Meves, 2014 & 0.870 & 0.789 & 0.923 & \\
Pohl, 2013 & 0.880 & 0.771 & 0.941 & \\
Akerman, 2012 & 0.970 & 0.818 & 0.996 & \\
Kalaitzakis, 2012 & 0.950 & 0.907 & 0.974 & \\
Draganov, 2011 & 0.933 & 0.849 & 0.972 & \\
Chen. 2011 & 0.983 & 0.960 & 0.993 & \\
Moon, 2009b & 0.780 & 0.606 & 0.891 & \\
Summary Rate & 0.913 & 0.876 & 0.940 & \\
Prediction Interval: & & \multicolumn{3}{|c}{} \\
\hline
\end{tabular}

Fig. 4 Forest plot of studies reporting technical success rate of peroral cholangioscopy for stone-related indications. Pooled success rate was $91 \%$ (95\% confidence interval $[\mathrm{Cl}] 88 \%-94 \%$ ). 


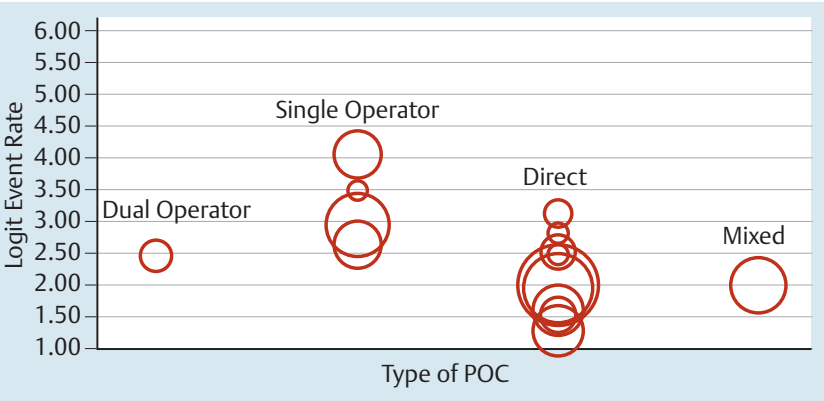

Fig. 5 Relationship between technical success rate for stone-related indications and type of peroral cholangioscopy (POC). Single-operator catheter-based cholangiography had a higher rate of technical success for stonerelated indications compared with other methods.

type of POC used and visual sensitivity ( $P=0.01)$, with dual-operator cholangioscopy having higher rates compared with SOC. There was a potential trend toward an association between the number of biopsies and accuracy $(P=0.077)$ such that an increased number of biopsies was associated with increased accuracy. The estimated technical success rate $(\mathrm{n}=18$ studies) was $94 \%$ (95\%Cl 90\%-96\%) ( Fig. 8), with significant evidence of heterogeneity $\left(P<0.011, I^{2}=67.39\right)$.

\section{Adverse events of peroral cholangioscopy}

The estimated overall adverse event rate was 7\% (95\%CI 6\%-9\%) $(\bullet$ Fig.9). The estimated rates of pancreatitis, cholangitis, perforation, and other adverse events were $2 \%$ (95\%CI $2 \%-3 \%$ ), $4 \%$ (95\%CI $3 \%-5 \%), 1 \%(95 \% \mathrm{CI} 1 \%-2 \%)$, and 3\% (95\%CI $2 \%-4 \%$ ), respectively. The estimated rate of severe adverse events was $1 \%$ (95\%CI $1 \%-2 \%$ ).
Study

Event rate and $95 \% \mathrm{Cl}$

\begin{tabular}{llll|l|l}
\hline & $\begin{array}{l}\text { Event } \\
\text { rate }\end{array}$ & $\begin{array}{l}\text { Lower } \\
\text { limit }\end{array}$ & $\begin{array}{l}\text { Upper } \\
\text { limit }\end{array}$ & \\
Chen, 2011 & 0.800 & 0.708 & 0.869 & & \\
Hartmann, 2012 & 0.870 & 0.598 & 0.968 & & \\
Tischendorf, 2006 & 0.930 & 0.822 & 0.974 & & \\
Alameel, 2013 & 0.840 & 0.606 & 0.947 & & \\
Chen, 2007 & 0.850 & 0.624 & 0.951 & & \\
Fukuda, 2005 & 0.934 & 0.851 & 0.972 & & \\
Nishikawa, 2013 & 0.970 & 0.814 & 0.996 & & \\
Osanai, 2013 & 0.921 & 0.782 & 0.974 & & \\
Ramchandani, 2011 & 0.890 & 0.740 & 0.958 & & \\
Woo, 2014 & 0.967 & 0.803 & 0.995 & & \\
Summary Rate & 0.894 & 0.844 & 0.929 & & \\
Prediction Interval: & & & & $\mathbf{0 . 0 0}$ & $\mathbf{0 . 5 0}$ \\
\hline
\end{tabular}

Fig. 6 Forest plot of studies reporting visual accuracy of peroral cholangioscopy in diagnosing indeterminate biliary strictures. Pooled accuracy rate was $89 \%$ ( $95 \%$ confidence interval $[\mathrm{Cl}] 84 \%-93 \%$ ).

\section{Discussion}

\section{$\nabla$}

POC has become a valuable tool for the treatment of difficult bile duct stones and the evaluation of indeterminate strictures. Despite increasing clinical use, there are very limited composite data evaluating its efficacy and safety. The aims of this study were to systematically review and analyze the efficacy of POC for difficult bile duct stones and indeterminate biliary strictures. The results of this systematic review and meta-analysis demonstrate a high stone clearance rate with the use of POC for difficult bile duct stones ( $88 \%$, 95\%CI $85 \%-91 \%)$. Similarly, POC showed an accuracy of $89 \%$ ( $95 \%$ CI $84 \%$ - $93 \%$ ) for visual impression of indeterminate biliary strictures and of $79 \%$ (95\%CI $74 \%-84 \%$ ) for directed tissue sampling. Finally, POC was noted to have an overall low adverse event rate ( $7 \%, 95 \% \mathrm{Cl} 6 \%-9 \%)$.

This analysis found that the accuracy of the visual impression was greater than biopsy-related accuracy, likely because of the high

Table 3 Efficacy and safety of peroral cholangioscopy for the removal of bile duct stones and the diagnosis of indeterminate strictures.

\begin{tabular}{|c|c|c|c|c|c|}
\hline & Estimated & $95 \% \mathrm{Cl}$ & $P^{2}$ & $\begin{array}{l}\text { Heterogeneity? } \\
\text { (P value) }\end{array}$ & $\begin{array}{l}\text { Publication bias? } \\
\text { ( } P \text { value) }\end{array}$ \\
\hline \multicolumn{6}{|l|}{ Stones } \\
\hline Clearance rate & $88 \%$ & $85 \%-91 \%$ & 26.14 & No (0.09) & Yes (0.05) \\
\hline Recurrence rate & $13 \%$ & $7 \%-20 \%$ & 40.09 & No $(0.14)$ & No $(0.56)$ \\
\hline Technical success rate & $91 \%$ & $88 \%-94 \%$ & 61.72 & Yes $(<0.01)$ & No $(0.32)$ \\
\hline \multicolumn{6}{|l|}{ Strictures } \\
\hline Visual accuracy & $89 \%$ & $84 \%-93 \%$ & 35.21 & No $(0.13)$ & Yes $(0.01)$ \\
\hline Visual sensitivity & $93 \%$ & $85 \%-97 \%$ & 38.46 & No $(0.11)$ & Yes $(<0.01)$ \\
\hline Visual specificity & $85 \%$ & $79 \%-89 \%$ & 0 & No $(0.84)$ & No $(0.50)$ \\
\hline Biopsy accuracy & $79 \%$ & $74 \%-84 \%$ & 19.12 & No (0.09) & Yes $(0.01)$ \\
\hline Biopsy sensitivity & $69 \%$ & $57 \%-78 \%$ & 97.97 & Yes $(<0.01)$ & No $(0.07)$ \\
\hline Biopsy specificity & $94 \%$ & $89 \%-97 \%$ & 0 & No $(0.88)$ & No $(0.18)$ \\
\hline Technical success rate & $94 \%$ & $90 \%-96 \%$ & 67.39 & Yes $(<0.01)$ & Yes $(<0.01)$ \\
\hline \multicolumn{6}{|l|}{ Adverse event rate } \\
\hline Overall & $7 \%$ & $6 \%-9 \%$ & 32.36 & Yes $(0.02)$ & Yes $(<0.01)$ \\
\hline Pancreatitis & $2 \%$ & $2 \%-3 \%$ & 0 & No $(0.99)$ & Yes $(<0.01)$ \\
\hline Cholangitis & $4 \%$ & $3 \%-5 \%$ & 25.55 & No $(0.06)$ & Yes $(<0.01)$ \\
\hline Perforation & $1 \%$ & $1 \%-2 \%$ & 0 & No (0.99) & No $(0.73)$ \\
\hline Other events & $3 \%$ & $2 \%-4 \%$ & 37.74 & Yes $(0.01)$ & Yes $(<0.01)$ \\
\hline Serious events & $1 \%$ & $1 \%-2 \%$ & 0 & No (0.99) & No $(0.28)$ \\
\hline
\end{tabular}

$\mathrm{Cl}$, confidence interval. 


\section{Study}

Event rate and $95 \% \mathrm{Cl}$

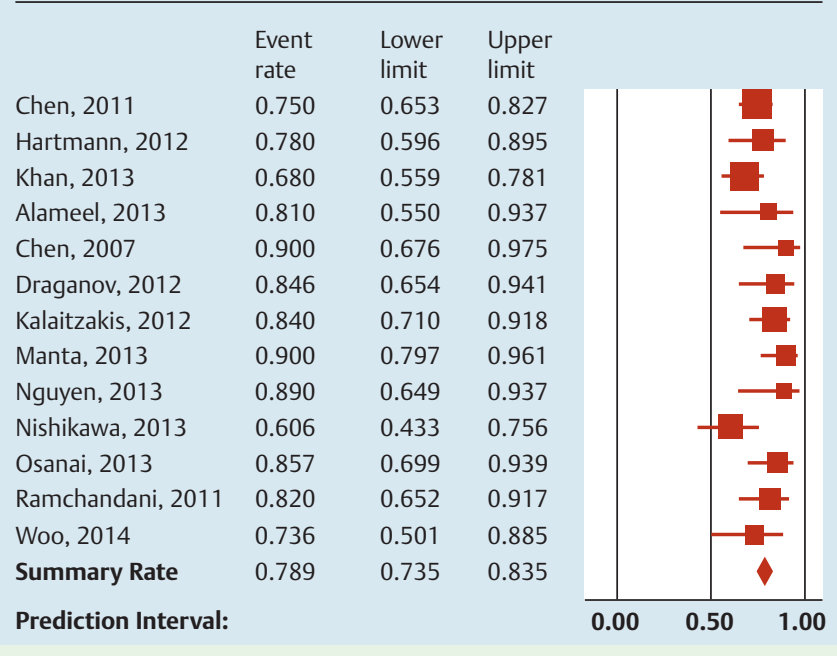

Fig. 7 Forest plot of studies reporting biopsy accuracy of peroral cholangioscopy in diagnosing indeterminate biliary strictures. Pooled accuracy rate was $79 \%$ (95\% confidence interval $[\mathrm{Cl}] 74 \%-94 \%$ ).

sensitivity of visual impression and poor sensitivity of biopsies. Currently, there is no standardized classification system used to help make a visual diagnosis of malignancy. However, studies evaluating POC for visual impression used characteristics such as the presence of irregular mucosa, an intraductal mass, or a tumor vessel to qualify a lesion as malignant, as these findings are often suggestive of malignancy $[9,14,20,43,44,48,53,56]$. It should be noted, however, that the data on the diagnostic characteristics of these individual characteristics are limited at the present time. Given the low specificity of visual impression, it cannot be used alone to confirm a diagnosis. This analysis also found that SOC systems had a significantly reduced sensitivity for visual impression when compared with dual-operator cholangioscopes. This is likely due to the fact that SOC systems provide a fiberoptic image that is of poorer quality than the digital image obtained with dual-operator cholangioscopes.

The suboptimal biopsy-related accuracy of POC was attributed to low overall sensitivity. This highlights the technical challenges of sampling indeterminate biliary strictures and calls for an improvement in tissue acquisition techniques. Our analysis found a statistically insignificant but potential trend toward greater accuracy with an increased number of biopsies. As suggested by Kalaitzakis et al. [29], taking more biopsy samples may result in an increased sensitivity (and potentially accuracy) for making a histological diagnosis. The high sensitivity of visual impression and high specificity of POC-directed biopsy make a combined approach, rather than the individual use of each, likely the most helpful method for making a diagnosis of malignancy.

Two meta-analyses $[58,59]$ have assessed the efficacy and diagnostic performance of SOC for indeterminate biliary strictures. One study [58] concluded that visual impression is useful for detecting a malignant lesion, and the other [59] that SOC biopsies have a moderate sensitivity for diagnosing malignant strictures. Both studies revealed that SOC is useful in confirming a malignant diagnosis because of its high specificity. One notable difference in this meta-analysis is that the studies involved looked at all types of POC and were not limited to SOC. However, the data from this meta-analysis are in concordance with those of the aforementioned meta-analyses in that they reveal a high sensi-

\begin{tabular}{llll|l} 
Study & & & \multicolumn{3}{c}{ Event rate and 95\% Cl } \\
\hline & $\begin{array}{l}\text { Event } \\
\text { rate }\end{array}$ & $\begin{array}{l}\text { Lower } \\
\text { limit }\end{array}$ & $\begin{array}{l}\text { Upper } \\
\text { limit }\end{array}$ \\
Akerman, 2012 & 0.970 & 0.818 & 0.996 & \\
Albert, 2011 & 0.880 & 0.687 & 0.961 \\
Chen, 2011 & 0.983 & 0.960 & 0.993 & \\
Farnik, 2014 & 0.885 & 0.818 & 0.930 \\
Itoi, 2014 & 0.830 & 0.684 & 0.917 & \\
Moon, 2009 & 0.780 & 0.606 & 0.891 & \\
Pohl, 2013 & 0.880 & 0.771 & 0.941 & \\
Tischendorf, 2006 & 0.991 & 0.869 & 0.999 & \\
Draganov, 2012 & 0.981 & 0.756 & 0.999 & \\
Draganov, 2011 & 0.933 & 0.849 & 0.972 & \\
Fukuda, 2005 & 0.995 & 0.924 & 1.000 & \\
Kalaitzakis, 2012 & 0.950 & 0.907 & 0.974 & \\
Manta, 2013 & 0.991 & 0.699 & 0.939 & \\
Meves, 2014 & 0.870 & 0.789 & 0.923 & \\
Nguyen, 2013 & 0.947 & 0.706 & 0.993 & \\
Nishikawa. 2013 & 0.985 & 0.804 & 0.999 & \\
Osanai, 2013 & 0.994 & 0.916 & 1.000 & \\
Ramchandani, 2011 & 0.986 & 0.818 & 0.999 \\
Summary Rate & 0.939 & 0.904 & 0.962 & \\
Prediction Interval: & & & & $\mathbf{0 . 0 0}$ \\
& & & $\mathbf{0 . 5 0}$
\end{tabular}

Fig. 8 Forest plot of studies reporting technical success rate of peroral cholangioscopy for stricture-related indications. Pooled success rate was $94 \%$ ( $95 \%$ confidence interval $[\mathrm{Cl}] 90 \%-96 \%$ ).

tivity of visual impression for the detection of malignant strictures and a high specificity associated with biopsy that can be useful in the confirmation of a malignant diagnosis.

POC appears to be a relatively safe procedure with a very low rate of serious events $(1 \%, 95 \% \mathrm{CI} 1 \%-2 \%)$. The data obtained in this systematic review and meta-analysis provide point estimates of adverse events that may be used in discussions with patients before a procedure. Notably, the patients undergoing POC have failed ERCP; this may be because they have more difficult anatomy or unusual lesions that require more manipulation. As such, there is a component of selection bias when patients are chosen to undergo POC. A recent study [60], completed in Sweden based on a national registry, reported that the risk for intra- and postprocedural adverse events is significantly increased when a patient undergoes POC in conjunction with ERCP, as opposed to ERCP alone. However, the study also noted that in a multivariate analysis that adjusted for confounders, the risk for pancreatitis and cholangitis was not increased. Of note, a systematic survey evaluating the incidence rates of post-ERCP complications [61] revealed an ERCP complication rate of approximately $6.85 \%$, with a severe event rate of approximately $1.67 \%$. These figures are comparable with the adverse event rates for POC estimated in this meta-analysis. Overall, it is clear that further research and data comparing POC with ERCP alone or with EUS are needed to compare the rates of adverse events and determine whether there is an increased adverse event rate with POC.

Limitations to this analysis included study heterogeneity and variability in the type of POC used. The studies had various patient populations, and the procedures were completed by using various methods of POC as well as differing instruments within each method. Furthermore, interoperator variability cannot be accounted for. Also, the definition of adverse event varied from study to study and accounted only for what was reported by the authors of each study. For example, some studies documented minor bleeding and considered it an adverse event, whereas oth- 


\begin{tabular}{|c|c|c|c|c|c|}
\hline \multirow[t]{2}{*}{ Study } & \multicolumn{3}{|c|}{ Statisitics for each study } & \multirow[t]{2}{*}{ Event rate and $95 \% \mathrm{Cl}$} & \multirow{3}{*}{$\begin{array}{l}\text { Fig. } 9 \text { Forest plot of studies reporting overall ad- } \\
\text { verse event rates of peroral cholangioscopy. Pooled } \\
\text { event rate was } 7 \% \text { ( } 95 \% \text { confidence interval }[\mathrm{Cl}] \\
6 \%-9 \% \text { ). }\end{array}$} \\
\hline & $\begin{array}{l}\text { Event } \\
\text { rate }\end{array}$ & $\begin{array}{l}\text { Lower } \\
\text { limit }\end{array}$ & $\begin{array}{l}\text { Upper } \\
\text { limit }\end{array}$ & & \\
\hline Akerman, 2012 & 0.015 & 0.001 & 0.196 & & \\
\hline Alameel, 2013 & 0.067 & 0.017 & 0.231 & & \\
\hline Albert, 2011 & 0.045 & 0.006 & 0.261 & & \\
\hline Arya, 2004 & 0.181 & 0.115 & 0.272 & & \\
\hline Awadallah, 2006 & 0.055 & 0.018 & 0.156 & & \\
\hline Chen, 2007 & 0.063 & 0.016 & 0.218 & & \\
\hline Chen, 2011 & 0.072 & 0.047 & 0.108 & & \\
\hline Draganov, 2011 & 0.048 & 0.018 & 0.121 & & \\
\hline Draganov, 2012 & 0.077 & 0.019 & 0.261 & & \\
\hline Farnik, 2014 & 0.087 & 0.047 & 0.154 & & \\
\hline Farrell, 2005 & 0.005 & 0.000 & 0.068 & & \\
\hline Fishman, 2009 & 0.006 & 0.000 & 0.087 & & \\
\hline Fukuda, 2005 & 0.021 & 0.005 & 0.079 & & \\
\hline Huang, 2013 & 0.022 & 0.001 & 0.268 & & \\
\hline Itoi, 2010a & 0.120 & 0.071 & 0.196 & & \\
\hline Itoi, 2014 & 0.059 & 0.015 & 0.207 & & \\
\hline Itoi, 2012 & 0.020 & 0.001 & 0.251 & & \\
\hline Itoi, 2010b & 0.069 & 0.038 & 0.124 & & \\
\hline Jakobs, 2007 & 0.028 & 0.002 & 0.322 & & \\
\hline Kalaitzakis, 2012 & 0.094 & 0.058 & 0.147 & & \\
\hline Kim, 2011 & 0.083 & 0.012 & 0.413 & & \\
\hline Lee, TY, 2012 & 0.083 & 0.012 & 0.413 & & \\
\hline Lee, YN, 2012 & 0.011 & 0.001 & 0.149 & & \\
\hline Liu, 2014 & 0.015 & 0.001 & 0.196 & & \\
\hline Manta, 2013 & 0.038 & 0.010 & 0.141 & & \\
\hline Maydeo, 2011 & 0.133 & 0.068 & 0.245 & & \\
\hline Meves, 2014 & 0.138 & 0.080 & 0.227 & & \\
\hline Moon, 2009a & 0.028 & 0.002 & 0.322 & & \\
\hline Moon, 2009b & 0.019 & 0.001 & 0.244 & & \\
\hline Mori, 2012 & 0.013 & 0.001 & 0.178 & & \\
\hline Neuhaus, 1993 & 0.038 & 0.002 & 0.403 & & \\
\hline Nguyen, 2013 & 0.056 & 0.008 & 0.307 & & \\
\hline Nishikawa, 2013 & 0.061 & 0.015 & 0.212 & & \\
\hline Osanai, 2013 & 0.069 & 0.031 & 0.145 & & \\
\hline Patel, 2014 & 0.043 & 0.014 & 0.126 & & \\
\hline Piraka, 2007 & 0.038 & 0.010 & 0.141 & & \\
\hline Pohl, 2013 & 0.132 & 0.064 & 0.252 & & \\
\hline Ramchandani, 2011 & 0.083 & 0.027 & 0.229 & & \\
\hline Sauer, 2013 & 0.250 & 0.108 & 0.478 & & \\
\hline Sepe, 2012 & 0.028 & 0.002 & 0.322 & & \\
\hline Shah, 2006 & 0.056 & 0.021 & 0.139 & & \\
\hline Siddiqui, 2012 & 0.033 & 0.005 & 0.202 & & \\
\hline Tischendorf, 2006 & 0.009 & 0.001 & 0.131 & & \\
\hline Tsuyuguchi, 2000 & 0.021 & 0.001 & 0.259 & & \\
\hline Woo, 2014 & 0.097 & 0.032 & 0.261 & & \\
\hline Summary Rate & 0.073 & 0.059 & 0.089 & & \\
\hline Prediction Interval: & & & & 0.50 & \\
\hline
\end{tabular}

ers did not. It should also be noted that are various types of difficult stones - large stones, confluence stones, impacted stones, etc. Although the meta-regression found no association between the size and location of stones, confluence stones and impacted stones were not specifically addressed in most studies. Therefore, they could not be distinctly evaluated in this analysis. Finally, it is important to make a distinction between filling defects caused by malignant strictures and filling defects caused by extrinsic compression/factors. Unfortunately, information on the latter was often very limited and not made distinct in the literature. Thus, the use of POC for detecting malignancy in filling defects caused by external compression or other factors could not be analyzed in this study.
POC is a safe and effective adjunctive tool with ERCP for the evaluation of bile duct strictures and for the treatment of bile duct stones when conventional methods have failed. Despite the increasing utilization of POC and technical advances such as the recently introduced digital single-operator cholangioscope, the current systematic review and meta-analysis confirm the paucity of high level evidence supporting the use of POC. Prospective, controlled clinical trials are needed to further elucidate the precise role of POC and develop criteria that can be used to standardize the diagnosis and treatment of pancreaticobiliary diseases. 
Competing interests: V. Raman Muthusamy, MD, and Srinadh Komanduri, MD, are consultants for Boston Scientific.

\section{Institutions}

${ }^{1}$ Division of Gastroenterology and Hepatology, Northwestern University Feinberg School of Medicine, Chicago Illinois, USA

2 Division of Gastroenterology, University of Colorado School of Medicine,

Aurora, Colorado, USA

Division of Gastroenterology, California Pacific Medical Center, San Francisco, California, USA

${ }^{4}$ UCLA Division of Digestive Diseases, Los Angeles, California, USA

Gastrointestinal Oncology, H. Lee Moffitt Cancer Center \& Research Institute,

Tampa, Florida, USA

\section{References}

1 Brauer BC, Shah RJ. Cholangioscopy in liver disease. Clin Liver Dis 2014; 18: $927-944$

2 Moon JH, Terheggen G, Choi HJ et al. Peroral cholangioscopy: diagnostic and therapeutic applications. Gastroenterology 2013; 144: 276-282

3 Parsi MA. Peroral cholangioscopy in the new millennium. World J Gastroenterol 2011; 17: 1 -6

4 Waxman I, Chennat J, Konda V. Peroral direct cholangioscopic-guided selective intrahepatic duct stent placement with an ultraslim endoscope. Gastrointest Endosc 2010; 71: 875-878

5 Shah RJ. Cholangioscopy and pancreatoscopy. In: Howell DA, ed. UpToDate. Waltham, MA: UpToDate; Last updated August 3, 2015

6 Liberati A, Altman DG, Tetzlaff J et al. The PRISMA statement for reporting systematic reviews and meta-analyses of studies that evaluate health care interventions: explanation and elaboration. Ann Intern Med 2009; 151: W65-W94

7 Wells GA, Shea B, Peterson JEA et al. The Newcastle-Ottawa Scale (NOS) for assessing the quality of nonrandomised studies in meta-analyses. Ottawa, Ontario, Canada: Ottawa Hospital Research Institute; 2014

8 Akerman S, Rahman M, Bernstein DE. Direct cholangioscopy: the North Shore experience. Eur J Gastroenterol Hepatol 2012; 24: 1406 - 1409

9 Alameel T, Bain V, Sandha G. Clinical application of a single-operator direct visualization system improves the diagnostic and therapeutic yield of endoscopic retrograde cholangiopancreatography. Can J Gastroenterol 2013; 27: 15-19

10 Albert JG, Friedrich-Rust M, Elhendawy $M$ et al. Peroral cholangioscopy for diagnosis and therapy of biliary tract disease using an ultra-slim gastroscope. [Erratum appears in Endoscopy 2011; 43: 1009]. Endoscopy 2011; 43: 1004-1009

11 Arya N, Nelles SE, Haber GB et al. Electrohydraulic lithotripsy in 111 patients: a safe and effective therapy for difficult bile duct stones. Am J Gastroenterol 2004; 99: 2330-2334

12 Awadallah NS, Chen YK, Piraka C et al. Is there a role for cholangioscopy in patients with primary sclerosing cholangitis? Am J Gastroenterol 2006; 101: 284-291

13 Chen YK, Parsi MA, Binmoeller KF et al. Single-operator cholangioscopy in patients requiring evaluation of bile duct disease or therapy of biliary stones (with videos). Gastrointest Endosc 2011; 74: 805-814

14 Chen YK, Pleskow DK. SpyGlass single-operator peroral cholangiopancreatoscopy system for the diagnosis and therapy of bile-duct disorders: a clinical feasibility study (with video). Gastrointest Endosc 2007; 65: $832-841$

15 Draganov PV, Chauhan S, Wagh MS et al. Diagnostic accuracy of conventional and cholangioscopy-guided sampling of indeterminate biliary lesions at the time of ERCP: a prospective, long-term follow-up study. Gastrointest Endosc 2012; 75: 347 - 353

16 Draganov PV, Lin T, Chauhan S et al. Prospective evaluation of the clinical utility of ERCP-guided cholangiopancreatoscopy with a new direct visualization system. Gastrointest Endosc 2011; 73: 971 - 979

17 Farnik H, Weigt J, Malfertheiner P et al. A multicenter study on the role of direct retrograde cholangioscopy in patients with inconclusive endoscopic retrograde cholangiography. Endoscopy 2014; 46: 16-21

18 Farrell JJ, Bounds BC, Al-Shalabi S et al. Single-operator duodenoscopeassisted cholangioscopy is an effective alternative in the management of choledocholithiasis not removed by conventional methods, including mechanical lithotripsy. Endoscopy 2005; 37: 542 - 547

19 Fishman DS. Management of pancreaticobiliary disease using a new intra-ductal endoscope: the Texas experience. World J Gastroenterol 2009; 15: $1353-1358$
20 Fukuda Y, Tsuyuguchi T, Sakai Y et al. Diagnostic utility of peroral cholangioscopy for various bile-duct lesions. Gastrointest Endosc 2005; 62: $374-382$

21 Hartman DJ, Slivka A, Giusto DA et al. Tissue yield and diagnostic efficacy of fluoroscopic and cholangioscopic techniques to assess indeterminate biliary strictures. Clin Gastroenterol Hepatol 2012; 10: 1042 1046

22 Huang SW, Lin CH, Lee MS et al. Residual common bile duct stones on direct peroral cholangioscopy using ultraslim endoscope. World J Gastroenterol 2013; 19: 4966 - 4972

23 Itoi T, Reddy ND, Sofuni A et al. Clinical evaluation of a prototype multibending peroral direct cholangioscope. Dig 2014; 26: 100 - 107

24 Itoi T, Osanai M, Igarashi Yet al. Diagnostic peroral video cholangioscopy is an accurate diagnostic tool for patients with bile duct lesions. Clin Gastroenterol Hepatol 2010; 8: $934-938$

25 Itoi $T$, Sofuni A, Itokawa $F$ et al. Diagnostic and therapeutic peroral direct cholangioscopy in patients with altered GI anatomy (with videos). Gastrointest Endosc 2012; 75: 441 - 449

26 Itoi $T$, Sofuni A, Itokawa $F$ et al. Evaluation of residual bile duct stones by peroral cholangioscopy in comparison with balloon-cholangiography. Digestive Endoscopy 2010; 22: S85-S89

27 Jakobs R, Maier M, Kohler B et al. Peroral laser lithotripsy of difficult intrahepatic and extrahepatic bile duct stones: laser effectiveness using an automatic stone-tissue discrimination system. Am J Gastroenterol 1996; 91: $468-473$

28 Jakobs R, Pereira-Lima JC, Schuch AW et al. Endoscopic laser lithotripsy for complicated bile duct stones: is cholangioscopic guidance necessary? Arq Gastroenterol 2007; 44: 137 - 140

29 Kalaitzakis E, Webster GJ, Oppong KW et al. Diagnostic and therapeutic utility of single-operator peroral cholangioscopy for indeterminate biliary lesions and bile duct stones. Eur J Gastroenterol Hepatol 2012; 24: $656-664$

30 Khan AH, Austin GL, Fukami $N$ et al. Cholangiopancreatoscopy and endoscopic ultrasound for indeterminate pancreaticobiliary pathology. Dig Dis Sci 2013; 58: 1110-1115

31 Kim HI, Moon JH, Choi HJ et al. Holmium laser lithotripsy under direct peroral cholangioscopy by using an ultra-slim upper endoscope for patients with retained bile duct stones (with video). Gastrointest Endosc 2011; 74: 1127-1132

32 Lee TY, Cheon YK, Choe WH et al. Direct cholangioscopy-based holmium laser lithotripsy of difficult bile duct stones by using an ultrathin upper endoscope without a separate biliary irrigating catheter. Photomed Laser Surg 2012; 30: $31-36$

33 Lee YN, Moon JH, Choi HJ et al. Direct peroral cholangioscopy using an ultraslim upper endoscope for management of residual stones after mechanical lithotripsy for retained common bile duct stones. Endoscopy 2012; 44: 819-824

34 Liu R, Cox RnK, Siddiqui A et al. Peroral cholangioscopy facilitates targeted tissue acquisition in patients with suspected cholangiocarcinoma. Minerva Gastroenterol Dietol 2014; 60: 127-133

35 Manta R, Frazzoni M, Conigliaro R et al. SpyGlass single-operator peroral cholangioscopy in the evaluation of indeterminate biliary lesions: a single-center, prospective, cohort study. Surg Endosc 2013; 27: 1569 1572

36 Maydeo A, Kwek BE, Bhandari S et al. Single-operator cholangioscopyguided laser lithotripsy in patients with difficult biliary and pancreatic ductal stones (with videos). Gastrointest Endosc 2011; 74: 1308 - 1314

37 Meves V, Ell C, Pohl J. Efficacy and safety of direct transnasal cholangioscopy with standard ultraslim endoscopes: results of a large cohort study. Gastrointest Endosc 2014; 79: 88-94

38 Moon JH, Ko BM, Choi HJ et al. Intraductal balloon-guided direct peroral cholangioscopy with an ultraslim upper endoscope (with videos). Gastrointest Endosc 2009; 70: $297-302$

39 Moon JH, Ko BM, Choi HJ et al. Direct peroral cholangioscopy using an ultra-slim upper endoscope for the treatment of retained bile duct stones. Am J Gastroenterol 2009; 104: 2729-2733

40 Mori A, Ohashi N, Nozaki M et al. Feasibility of duodenal balloon-assisted direct cholangioscopy with an ultrathin upper endoscope. Endoscopy 2012; 44: 1037-1044

41 Neuhaus H, Hoffmann W, Zillinger $C$ et al. Laser lithotripsy of difficult bile duct stones under direct visual control. Gut 1993; 34: 415-421

42 Nguyen NQ Schoeman MN, Ruszkiewicz A. Clinical utility of EUS before cholangioscopy in the evaluation of difficult biliary strictures. Gastrointest Endosc 2013; 78: 868 - 874 
43 Nishikawa T, Tsuyuguchi T, Sakai Y et al. Comparison of the diagnostic accuracy of peroral video-cholangioscopic visual findings and cholangioscopy-guided forceps biopsy findings for indeterminate biliary lesions: a prospective study. Gastrointest Endosc 2013; 77: 219-226

44 Osanai M, Itoi T, Igarashi $Y$ et al. Peroral video cholangioscopy to evaluate indeterminate bile duct lesions and preoperative mucosal cancerous extension: a prospective multicenter study. Endoscopy 2013; 45: 635-642

45 Patel SN, Rosenkranz L, Hooks B et al. Holmium-yttrium aluminum garnet laser lithotripsy in the treatment of biliary calculi using single-operator cholangioscopy: a multicenter experience (with video). Gastrointest Endosc 2014; 79: 344-348

46 Piraka C, Shah RJ, Awadallah NS et al. Transpapillary cholangioscopydirected lithotripsy in patients with difficult bile duct stones. Clin Gastroenterol Hepatol 2007; 5: 1333-1338

47 Pohl J, Meves VC, Mayer G et al. Prospective randomized comparison of short-access mother-baby cholangioscopy versus direct cholangioscopy with ultraslim gastroscopes. Gastrointest Endosc 2013; 78: 609616

48 Ramchandani M, Reddy DN, Gupta R et al. Role of single-operator peroral cholangioscopy in the diagnosis of indeterminate biliary lesions: a single-center, prospective study. Gastrointest Endosc 2011; 74: 511 519

49 Sauer BG, Cerefice M, Swartz DC et al. Safety and efficacy of laser lithotripsy for complicated biliary stones using direct choledochoscopy. Dig Dis Sci 2013; 58: 253-256

50 Sepe PS, Berzin TM, Sanaka S et al. Single-operator cholangioscopy for the extraction of cystic duct stones (with video). Gastrointest Endosc 2012; 75: 206-210

51 Shah RJ, Langer DA, Antillon MR et al. Cholangioscopy and cholangioscopic forceps biopsy in patients with indeterminate pancreaticobiliary pathology. Clin Gastroenterol Hepatol 2006; 4: 219-225

52 Siddiqui AA, Mehendiratta $V$, Jackson $W$ et al. Identification of cholangiocarcinoma by using the Spyglass Spyscope system for peroral chol- angioscopy and biopsy collection. Clin Gastroenterol Hepatol 2012; 10: 466-471; quiz e48

53 Tischendorf IJ, Kruger M, Trautwein C et al. Cholangioscopic characterization of dominant bile duct stenoses in patients with primary sclerosing cholangitis. [Erratum appears in Endoscopy 2006; 38: 852]. Endoscopy 2006; 38: $665-669$

54 Tsuyuguchi T, Saisho H, Ishihara T et al. Long-term follow-up after treatment of Mirizzi syndrome by peroral cholangioscopy. Gastrointest Endosc 2000; 52: 639-644

55 Tsuyuguchi T, Sakai Y, Sugiyama $H$ et al. Long-term follow-up after peroral cholangioscopy-directed lithotripsy in patients with difficult bile duct stones, including Mirizzi syndrome: an analysis of risk factors predicting stone recurrence. Surg Endosc 2011; 25: 2179-2185

56 Woo YS, Lee JK, Oh SH et al. Role of SpyGlass peroral cholangioscopy in the evaluation of indeterminate biliary lesions. Dig Dis Sci 2014; 59: $2565-2570$

57 Duval S, Tweedie R. Trim and fill: a simple funnel-plot-based method of testing and adjusting for publication bias in meta-analysis. Biometrics 2000; 56: $455-463$

58 Sun X, Zhou Z, Tian J et al. Is single-operator peroral cholangioscopy a useful tool for the diagnosis of indeterminate biliary lesion? A systematic review and meta-analysis Gastrointest Endosc 2015; 82: 79 87

59 Navaneethan U, Hasan MK, Lourdusamy Vet al. Single-operator cholangioscopy and targeted biopsies in the diagnosis of indeterminate biliary strictures: a systematic review. Gastrointest Endosc 2015; 82: 79 87

60 Lubbe J, Arnelo $U$, Lundell $L$ et al. ERCP-guided cholangioscopy using a single-use system: nationwide register-based study of its use in clinical practice. Endoscopy 2015; 47: 802-807

61 Andriulli A, Loperfido $S$, Napolitano $G$ et al. Incidence rates of post-ERCP complications: a systematic survey of prospective studies. Am J Gastroenterol 2007; 102: 1781 -1788 